\title{
Chronic fluoxetine treatment of juvenile zebrafish (Danio rerio) does not elicit changes in basal cortisol levels and anxiety-like behavior in adulthood
}

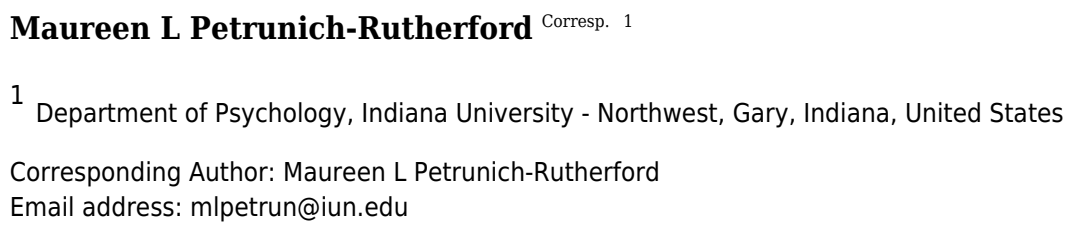

Exposure to selective serotonin reuptake inhibitors (SSRIs) during development may elicit long-term neuroadaptive changes that could alter the basal regulation of stress-associated physiological and behavioral processes later in life. Currently, the effects of juvenile fluoxetine exposure in rodent models appear to be dependent on the developmental window targeted as well as the duration of drug exposure. The zebrafish (Danio rerio) model is rapidly becoming a useful tool in pharmacological research and can be used to help elucidate some of the long-term effects of fluoxetine exposure prior to sexual maturation on neuroendocrine and behavioral stress markers. In the current study, juvenile zebrafish were chronically exposed to fluoxetine hydrochloride (0 or $100 \mu \mathrm{g} / \mathrm{L}$ ) for 14 days (31-44 days post-fertilization (dpf)), then were left untreated until young adulthood. Starting at $90 \mathrm{dpf}$, basal neuroendocrine stress and behavioral responses of zebrafish were assessed. Cortisol was extracted from the young adult zebrafish body (trunk) and quantified via ELISA. Anxiety-like behaviors were assessed in response to introduction to the novel tank test. It was expected that juvenile exposure to fluoxetine would (1) reduce basal cortisol levels and (2) elicit anxiolytic effects in the novel tank test in adulthood. However, fluoxetine exposure during the juvenile period was not associated with alterations in basal levels of cortisol nor were there any significant changes in anxiety-like behavior in the young adult zebrafish. Thus, in zebrafish, it does not appear that SSRI exposure during the juvenile period has a long-term adverse or maladaptive impact on the basal expression of cortisol and anxiety-like behavior in adulthood. Further studies are needed to determine if SSRI exposure during this developmental window influences neuroendocrine and behavioral responses to acute stress. 
1 Chronic fluoxetine treatment of juvenile zebrafish (Danio rerio) does not elicit

2 changes in basal cortisol levels and anxiety-like behavior in adulthood

3

4 Maureen L. Petrunich-Rutherford ${ }^{1}$

5

$6 \quad{ }^{1}$ Department of Psychology, Indiana University Northwest, Gary, Indiana, USA

7

8 Corresponding Author:

9 Maureen L. Petrunich-Rutherford ${ }^{1}$

10 Email address: mlpetrun@iun.edu

11

12 


\section{Abstract}

Exposure to selective serotonin reuptake inhibitors (SSRIs) during development may elicit long-term neuroadaptive changes that could alter the basal regulation of stress-associated physiological and behavioral processes later in life. Currently, the effects of juvenile fluoxetine exposure in rodent models appear to be dependent on the developmental window targeted as well as the duration of drug exposure. The zebrafish (Danio rerio) model is rapidly becoming a useful tool in pharmacological research and can be used to help elucidate some of the long-term effects of fluoxetine exposure prior to sexual maturation on neuroendocrine and behavioral stress markers. In the current study, juvenile zebrafish were chronically exposed to fluoxetine hydrochloride ( 0 or $100 \mu \mathrm{g} / \mathrm{L})$ for 14 days (31-44 days post-fertilization (dpf)), then were left untreated until young adulthood. Starting at $90 \mathrm{dpf}$, basal neuroendocrine stress and behavioral responses of zebrafish were assessed. Cortisol was extracted from the young adult zebrafish body (trunk) and quantified via ELISA. Anxiety-like behaviors were assessed in response to introduction to the novel tank test. It was expected that juvenile exposure to fluoxetine would

27 (1) reduce basal cortisol levels and (2) elicit anxiolytic effects in the novel tank test in adulthood.

28 However, fluoxetine exposure during the juvenile period was not associated with alterations in 29 basal levels of cortisol nor were there any significant changes in anxiety-like behavior in the young adult zebrafish. Thus, in zebrafish, it does not appear that SSRI exposure during the

31 juvenile period has a long-term adverse or maladaptive impact on the basal expression of cortisol 32 and anxiety-like behavior in adulthood. Further studies are needed to determine if SSRI exposure 33 during this developmental window influences neuroendocrine and behavioral responses to acute 34 stress. 


\section{Introduction}

Adolescence is a developmental time marked by tremendous amounts of brain growth and plasticity. However, it is also a period of particular mental vulnerability. It has been estimated that $12.5 \%$ of the U.S. population aged 12 to 17 - around 3 million adolescents - has had at least one episode of depression in the last year (Center for Behavioral Health Statistics and Quality, 2016). These depressive episodes can be isolated, or may be indicative of a chronic, relapsing condition that can be lifelong. Selective serotonin reuptake inhibitors (SSRIs) are currently the most effective pharmaceutical option for treating adolescent depressive and anxiety disorders (Ryan, 2003; Bostic et al., 2005; Gentile, 2010; Kodish, Rockhill \& Varley, 2011; Hetrick et al., 2012). Indeed, the SSRI fluoxetine (Prozac) is the only FDA-approved drug for treating childhood and adolescent depression, although other SSRIs are often prescribed on an “off-label” basis (“Antidepressant Medications," 2017). However, caution is still advised when prescribing antidepressants to youth, as the risk of increased suicidality or other negative side effects cannot be completely ruled out (Reeves \& Ladner, 2010; Cousins \& Goodyer, 2015; Vitiello \& Ordóñez, 2016). Additionally, in rodent models, chronic SSRI treatment prior to sexual maturation can elicit unique and/or long-lasting effects that can persist until adulthood, such as changes in the brain's serotonin-producing neurons (Maciag et al., 2006) and alterations in behavior (Ansorge et al., 2004; Oh et al., 2009; Iñiguez, Warren \& Bolaños-Guzmán, 2010; Iniguez et al., 2014). These unpredictable and unexpected changes in brain structure or function may potentially preclude the use of serotonergic antidepressants in adulthood, should depression be a chronic, lifelong condition. 
59 validated, low-cost, low-maintenance option for use in pharmacological and stress research due

60 to the conservation of mechanisms regulating biological responses to stress (for reviews, see

61 (Steenbergen, Richardson \& Champagne, 2011; Stewart et al., 2014). The neuroendocrine stress

62 axis in teleost species (such as zebrafish), the hypothalamic-pituitary-interrenal (HPI) axis, is

63 analogous to the hypothalamic-pituitary-adrenal (HPA) axis of mammals (Wendelaar Bonga,

64 1997; Nesan \& Vijayan, 2013). As observed in rodents, SSRIs can also alter stress responses in

65 zebrafish. For example, acute exposure to fluoxetine in adult zebrafish suppresses cortisol

66 responses (Abreu et al., 2014, 2015, 2017), increases extracellular levels of brain serotonin

67 (Maximino et al., 2014), and alters behavioral responses (Maximino et al., 2014; Giacomini et

68 al., 2016a) observed in response to acute stress exposure. Paradoxically, however, acute

69 fluoxetine exposure in the absence of environmental stressors is associated with increased

70 cortisol responses and alterations in brain mRNA levels for stress-axis related transcripts

71 (Theodoridi, Tsalafouta \& Pavlidis, 2017). Additionally, acute fluoxetine exposure in the

72 absence of stress has been found to elicit both anxiogenic and anxiolytic effects on behavior,

73 depending on the type of test used (Maximino et al., 2013; Magno et al., 2015). Although acute

74 fluoxetine exposure studies are important for elucidating the early neural and behavioral

75 responses to drug exposure, SSRIs are typically used chronically; therefore, chronic treatment

76 paradigms in non-human animals are essential for understanding long-term neuroadaptive or

77 behavioral effects elicited in response to putative serotonergic alterations. Indeed, studies

78 involving chronic exposure of fluoxetine show more consistent results compared to studies

79 investigating acute exposure in zebrafish. Chronic fluoxetine treatment of adult zebrafish

80 induces anxiolytic effects in behavioral tests (Maximino et al., 2011; Wong, Oxendine \& 
81 Godwin, 2013), alters whole-brain gene expression of some stress-related genes (Wong,

82 Oxendine \& Godwin, 2013), blunts chronic stress-induced increases in cortisol levels (Giacomini

83 et al., 2016b; Marcon et al., 2016), and attenuates chronic stress-induced anxiogenic behaviors

84 and the whole-brain expression of gene markers of inflammation (Marcon et al., 2016).

The effects of fluoxetine are likely dependent on a host of factors, including the time and

86

87

88

89

90

91

92

93

94

95

96

97

duration of drug exposure, whether the dependent measures are assessed after a stressor or not, etc. Developmental stage is also crucial for understanding the impact of serotonergic modulation on long-term outcomes (Kiryanova, McAllister \& Dyck, 2013), including the development and expression of HPA/HPI neuroendocrine responses and anxiety-like behaviors. Exposure to fluoxetine during the embryonic stage affects mortality and growth (Kalichak et al., 2016). Additionally, fluoxetine exposure during the embryonic and larval periods alters gene expression (Park et al., 2012; Cunha et al., 2016; Crago \& Klaper, 2018), particularly of serotonergic system-related genes (Pei et al., 2017; Cunha et al., 2018) and those related to the HPI axis (Kwan et al., 2016). Although acute larval exposure to fluoxetine does not affect thigmotaxis (edge preference), a measure of anxiety-like behavior (Richendrfer et al., 2012), long-term effects of fluoxetine on other behaviors are dependent on the duration of time and specific developmental stage of drug exposure. For example, when zebrafish were exposed to fluoxetine for 24 hours starting on 3 days post-fertilization ( $\mathrm{dfp}$ ), only transient effects on motor activity were observed. However, the same fluoxetine treatment initiated on 4 dfp elicited effects on motor activity that persisted up to $14 \mathrm{dpf}$ (Airhart et al., 2007).

Few, if any, studies have examined the impact of developmental SSRI exposure on adult anxiety-like behavior or basal neuroendocrine markers in the zebrafish. Studies in rodents and primates have indicated that serotonin plays a role in the early life programming and function of 
104 the HPA axis, possibly by inducing permanent upregulation of the glucocorticoid receptor 105 (Andrews \& Matthews, 2004) or the serotonin transporter (Shrestha et al., 2014). It is possible 106 that developmental SSRI exposure in zebrafish may elicit long-term changes in the regulation of

107 the HPI axis as well; however, no studies have examined the impact of chronic exposure to 108 SSRIs during the juvenile period. The juvenile period (approximately 30-89 dpf) occurs after 109 the embryonic and larval periods, but prior to sexual maturation in zebrafish (Kalueff, Stewart \& 110 Gerlai, 2014). During the juvenile period, exposure to a mild daily stress plus environmental 111 enrichment decreases anxiety-like behavior in adulthood (DePasquale et al., 2016). However, it 112 is not known whether chronic fluoxetine exposure during the early juvenile period alters the 113 expression of anxiety-like behavior or basal levels of stress hormone responses during adulthood 114 in the zebrafish model.

115 In the current study, young juvenile zebrafish were chronically exposed to fluoxetine for 11614 days and assessed for basal cortisol levels and anxiety-like behavior in adulthood. Anxiety117 like behavior was assessed by the novel tank test, a well-validated measure of habituation to 118 novelty. Zebrafish tend to freeze and seek safe zones (e.g., the bottom of the novel tank) when 119 under threat. However, zebrafish also have an innate tendency to explore. Thus, a fish that is 120 demonstrating anxiety-like behavior will be less likely to explore the top of the tank. Based on 121 previously published studies in adult zebrafish, the hypothesis for the current study is that 122 juvenile exposure to fluoxetine will (1) reduce basal cortisol levels and (2) elicit anxiolytic 123 behaviors (e.g., increased exploratory behavior in the top of the novel tank) in adulthood. This 124 study is timely, as the potential impact of juvenile fluoxetine exposure on adult behavior and 125 neuroendocrine responses remains to be fully elucidated. 
127 Materials \& Methods

128 Animals. Wild-type zebrafish (Danio rerio) embryos were obtained from the Zebrafish

129 International Resource Center (ZIRC), Eugene, OR. All procedures for raising, feeding, and

130 using zebrafish were carried out by following established recommendations (Westerfield, 2000;

131 Harper \& Lawrence, 2011). Larval zebrafish (from hatching until 14 days post-fertilization (dpf))

132 were maintained in stagnant water at room temperature, fed twice daily with dried,

133 commercially-available larval fish food, and were subject to gentle water exchanges once daily.

134 On $15 \mathrm{dpf}$, fish were gently moved to the system, a two-shelf, stand-alone housing rack

135 (Aquaneering, San Diego, CA) with a slow drip. The drip was slowly increased every few days

136 to acclimate the fish to a steady stream of water by $30 \mathrm{dpf}$. The system was maintained on a

137 14:10 hour light/dark cycle, water temperature of $26 \pm 2{ }^{\circ} \mathrm{C}$, and $\mathrm{pH} 7.4 \pm 0.2$. After $30 \mathrm{dpf}$, fish

138 were fed once daily with commercially-available flake food.

139 Juvenile fluoxetine exposure. Starting at $31 \mathrm{dpf}$, zebrafish (mixed sexes) were randomly

140 moved to one of two $1.8 \mathrm{~L}$ housing tanks. Each housing tank was assigned at random to either 0

141 or $100 \mu \mathrm{g} / \mathrm{L}$ fluoxetine hydrochloride (Santa Cruz Biotechnology, Inc., Dallas, TX). The dose,

142 time, and route of drug administration was based on previously published studies (Egan et al.,

143 2009; Wong, Oxendine \& Godwin, 2013; Pittman \& Lott, 2014; Pittman \& Hylton, 2015).

144 Fluoxetine was prepared as a concentrated stock solution $(0.5 \mathrm{mg} / \mathrm{ml}$ system water $)$, then, daily,

145 was diluted to the final concentration of $100 \mu \mathrm{g} / \mathrm{L}$ in $1 \mathrm{~L}$ system water in a clear rectangular

146 polycarbonate container with lid. Fish in the control condition were handled the same as the

147 fluoxetine-treated fish; however, fish were placed in 1L system water in a separate container.

148 The fish were gently transferred by net from their home tank into the appropriate treatment

149 container for one hour per day for 14 days (modified from Pittman \& Hylton, 2015). After the 
150 daily treatment, fish were placed back into clean home tanks, which were then placed back in the

151 system. Fresh treatment and control tanks were prepared each day of the chronic treatment.

152 After the treatment, fish were allowed to mature to adulthood in their home tanks without further

153 treatment or handling other than daily maintenance. The sample size of the groups was chosen

154 based on the effect elicited by chronic fluoxetine in adults (Marcon et al., 2016) and was

155 sufficient to give the study $>80 \%$ statistical power (as per Anderson, Kelley \& Maxwell, 2017);

156 https://designingexperiments.com/shiny-r-web-apps/).

157 Novel tank test. Before the data collection (starting on $90 \mathrm{dpf}$ ), both housing tanks were

158 removed from the system and brought into the procedural room and allowed to acclimate for at

159 least 30 minutes. Individual fish were selected at random, netted, and placed into a trapezoidal

160 novel tank (approximately 3"x13"x6", Aquaneering part number ZT180T) for six minutes. The

161 behavior of the fish were recorded and subsequently analyzed with Ethovision XT motion-

162 tracking software (Noldus, Leesburg, VA). Number of entries to the top of tank, time spent in

163 top $(\mathrm{sec})$, distance traveled in the top $(\mathrm{cm})$, latency to enter the top $(\mathrm{sec})$, frequency of freezing,

164 and freezing duration ( $\mathrm{sec}$ ) were used as markers of anxiety-like behavior. A fish that

165 demonstrates anxiety-like behavior will be less likely to explore the top of the tank and will show

166 more freezing behavior. Total distance traveled $(\mathrm{cm})$ and mean speed $(\mathrm{cm} / \mathrm{sec})$ were measured

167 as controls to ensure the chronic treatment did not compromise activity levels (Cachat et al., 168 2010).

169 Euthanasia and dissection. Fifteen minutes after introduction to the novel tank test, each

170 fish was placed individually in a $50 \mathrm{ml}$ beaker containing $30 \mathrm{ml}$ of $0.1 \%(100 \mathrm{mg} / \mathrm{L})$ clove oil in

171 system water. Death was determined upon visual examination for cessation of opercular (gill)

172 movement and non-response to tactile stimulation (Davis et al., 2015). The fish were then 
173 decapitated; each trunk was placed in a microcentrifuge tube and stored at $-20^{\circ} \mathrm{C}$ until cortisol

174 extraction and analysis.

175 Determination of trunk cortisol. Trunk samples were used for assessing levels of cortisol

176 (as per Cachat et al., 2010; Canavello et al., 2011). Briefly, trunk samples were thawed and

177 weighed, then homogenized in $1 \mathrm{ml}$ ice-cold $25 \mathrm{mM}$ phosphate-buffered saline (PBS) solution.

178 To extract the cortisol, diethyl ether $(5 \mathrm{ml})$ was added to the homogenates. After centrifugation

179 at $4^{\circ} \mathrm{C}$ for 15 minutes at $2500 \mathrm{rpm}$, the organic layer containing the cortisol was transferred to a

180 new test tube. This procedure was repeated three times in order to maximize the extracted

181 cortisol from the homogenates. The volatile compounds were allowed to evaporate under a

182 hood. After the evaporation, $1 \mathrm{ml} 25 \mathrm{mM}$ PBS was added to the lipid layer containing cortisol

183 left in each tube. To determine cortisol levels, a cortisol ELISA was used (Salimetrics, State

184 College, PA) as per the manufacturer's instructions. Cortisol levels were determined by

185 comparing ELISA binding values to a standard curve. Cortisol values were normalized to trunk

186 weight; the final cortisol data are expressed as ng cortisol/g trunk weight.

187 Data analysis. Each fish was given a sample number; behavioral and cortisol data were

188 not grouped by treatment until final behavioral measures and cortisol values were obtained. At

189 the end of the experiment, there was a total of $n=17$ for the control group and $n=21$ for the

190 fluoxetine-treated group. Values were excluded from analyses if two standard deviations above

191 or below the mean for each assay. For the cortisol assay, a total of four samples (two samples

192 from each treatment group) were removed from analyses either due to issues with the extraction

193 procedure $(\mathrm{n}=3)$ or because the value was identified as an outlier in its respective treatment

194 group $(n=1)$. For the behavioral analysis, several fish $(n=4$ in the control group and $n=9$ in

195 the fluoxetine-treated group) were removed from analyses due to one or more behavioral 
196 parameters falling outside the two standard deviation threshold. Data are presented as group

197 means and the standard errors of the mean (SEM). All data were analyzed by independent

198 samples t tests. JASP software (https://jasp-stats.org/) was used for all statistical analyses. A

199 significance value of $\mathrm{p}<0.05$ was used as the criterion for a result to reach statistical

200 significance.

201

202 Results

203

Chronic juvenile fluoxetine treatment did not alter trunk cortisol responses. Trunk

204

205

206

207

208

209

210

211

212

213

214

215

216

217

218

cortisol levels of zebrafish chronically treated with fluoxetine prior to maturation did not differ

from control fish (figure 1). An independent samples $t$ test indicated no significant effect of treatment on trunk cortisol levels $(\mathrm{t}(23)=-0.027, \mathrm{p}=0.979)$.

Chronic juvenile fluoxetine treatment did not alter motor activity of zebrafish in the novel

tank test. Measures of motor activity (total distance moved $(\mathrm{cm})$ and mean speed $(\mathrm{cm} / \mathrm{sec})$ ) did not differ in zebrafish chronically treated with fluoxetine prior to maturation compared to control fish (figure 2). An independent samples $t$ test indicated no significant effect of treatment on total distance moved $(\mathrm{t}(23)=-1.053, \mathrm{p}=0.303)$ and no significant effect of treatment on mean speed $(t(23)=-0.540, p=0.594)$.

Chronic juvenile fluoxetine treatment did not alter freezing behavior of zebrafish in the novel tank test. Measures of freezing (number of immobile bouts and total time spent immobile (s)) did not differ in zebrafish chronically treated with fluoxetine prior to maturation compared to control fish (figure 3). An independent samples $t$ test indicated no significant effect of treatment

217 on the number of times zebrafish were immobile $(\mathrm{t}(23)=0.482, \mathrm{p}=0.634)$ and no significant

218 effect of treatment on the time spent immobile $(\mathrm{t}(23)=-0.539, \mathrm{p}=0.595)$. 
novel tank test. Measures of anxiety-like behavior (distance in top (cm), number of entries to

\section{1}

top, total time in top (s), and latency to top (s)) did not differ in zebrafish chronically treated with

222

223

224

225

226

227

228

229

230

231

232

233

234

235

236

237

238

239

240

241

fluoxetine prior to maturation compared to control fish (figure 4). An independent samples t test indicated no significant effect of treatment on the distance traveled in the top of the novel tank $(\mathrm{t}(23)=0.376, \mathrm{p}=0.710)$, no significant effect of treatment on the number of times zebrafish traversed to the top of the novel tank $(\mathrm{t}(23)=-0.195, \mathrm{p}=0.847)$, no significant effect of treatment on the total time spent in the top of the novel tank $(\mathrm{t}(23)=0.500, \mathrm{p}=0.622)$, and no significant effect of treatment on the latency to enter the top $(t(23)=0.020, p=0.984)$.

\section{Discussion}

The present study is the first to investigate the long-term effects of juvenile fluoxetine exposure on adult markers of basal stress regulation in zebrafish. Exposure to fluoxetine for 14 days during the juvenile period (31-44 dpf) was not associated with significant alterations in basal levels of cortisol or indicators of anxiety-like behavior. Thus, the results of the current study suggest that juvenile zebrafish are resilient to or overcome any SSRI-induced neuroadaptations at this dose and time of fluoxetine exposure, at least concerning the basal regulation of the stress response pathway and expression of anxiety-like behavior. These results are consistent with at least one other study in rodents that demonstrated that fluoxetine exposure during adolescence was not associated with increased anxiety-like behavior in adulthood (Norcross et al., 2008). Although some other rodent studies have demonstrated fluoxetineinduced alterations in adult anxiety-like behavior, this could be due, in part, to the timing of the developmental drug exposure. Earlier exposure, such as during the rodent prepubertal period 
242 (e.g., around postnatal day 21, as targeted in Ansorge et al., 2004; Oh et al., 2009) may elicit

243 some changes in still-maturing brain pathways that could be more resilient to change slightly

244 later in development. Additional studies done in zebrafish may help to clarify the impact of

245 manipulating serotonin levels at specific developmental stages (e.g., larval, early juvenile, late 246 juvenile) on stress-related markers in adulthood.

247 Chronic fluoxetine treatment did not affect the long-term regulation of basal levels of 248 stress hormones or behavioral responses during adulthood in the current study. The sample size 249 of the groups in the current study is consistent with other previously published reports that 250 demonstrate that chronic fluoxetine significantly impacts a variety of biological and behavioral 251 markers in adult zebrafish (Maximino et al., 2011; Wong, Oxendine \& Godwin, 2013; Giacomini 252 et al., 2016b; Marcon et al., 2016). Additionally, a power analysis indicated that the sample size 253 used in the current study would have been sufficient to detect an equivalent size effect as elicited 254 by chronic fluoxetine exposure in adults. Thus, if there is any effect of developmental exposure 255 to fluoxetine, it has a much smaller impact on basal neuroendocrine responses and anxiety-like 256 behavior compared to adult fluoxetine exposure. Further studies examining fluoxetine exposure 257 in developing vs. adult fish in the same cohort would be necessary to verify the magnitude of 258 differences between these two exposure paradigms.

259 Additionally, this experiment measured basal levels of stress responses elicited by 260 introduction to a novel tank. The addition of a stronger acute stressor, such as net chasing 261 (Abreu et al., 2014; Giacomini et al., 2016a,b), may reveal some subtle fluoxetine-induced 262 effects on the expression of stress-induced neuroendocrine and behavioral responses compared to 263 those induced by transfer into a novel tank. Furthermore, in this study, males and females were 264 pooled. Future studies should determine if there are any sex-dependent differences in fluoxetine- 
265 induced alterations in stress responses, as other studies have demonstrated sex-dependent 266 differences in stress regulation in response to exposure to chronic unpredictable stress in 267 zebrafish (Rambo et al., 2017) and to fluoxetine exposure in rodents (Pawluski et al., 2012).

268 Another possible explanation for the results of the current study involve the route of drug 269 administration. Daily handling involved with administering fluoxetine in the current design may 270 have served as a mild stressor and, thus, induced resiliency in the subjects in adulthood. Thus, 271 any fluoxetine-induced alterations may have been overshadowed by the effects of daily handling 272 during the juvenile period. In a previously published study, a mild daily stressor plus 273 environmental enrichment during the juvenile period was associated with decreased anxiety 274 behavior in adulthood (DePasquale et al., 2016). Additional studies would be necessary to 275 determine whether handling alone has an impact on the adult expression of anxiety-like behavior 276 and basal stress hormone responses in zebrafish. Additionally, in this study, young adult 277 zebrafish (approximately $90 \mathrm{dpf}$ ) were exposed to zero or $100 \mu \mathrm{g} / \mathrm{L}$ fluoxetine. This dose was 278 chosen based on previous literature using adults (Egan et al., 2009; Wong, Oxendine \& Godwin, 279 2013). Future studies using a range of doses would be necessary to fully elucidate the full 280 impact of juvenile fluoxetine exposure on adult stress-associated behaviors and neuroendocrine 281 responses (Stewart et al., 2014; Sumpter, Donnachie \& Johnson, 2014).

282 Young adult zebrafish in the current study were sacrificed at fifteen minutes post283 introduction to the novel tank test in order to assess cortisol levels. This time point was chosen 284 due to previous research demonstrating that the peak cortisol response occurs at least 15 minutes 285 post-stressor in zebrafish (Ramsay et al., 2009; Abreu et al., 2014; Tran, Chatterjee \& Gerlai, 286 2014; Pavlidis, Theodoridi \& Tsalafouta, 2015). However, the peak cortisol response could have 287 occurred at an earlier or later time point in young adult fish compared to older adult zebrafish. It 
288 is possible that young adult zebrafish may have an immature or alternative time course for 289 cortisol release. There is some evidence of possible age-dependent differences in whole-body 290 cortisol at young vs. older adult stages in zebrafish (Ramsay et al., 2009), yet no published 291 studies have established a full cortisol time course of young (90 dpf) adult fish. With regards to 292 the results of the current study, however, the lack of significant basal cortisol alterations in 293 fluoxetine-treated fish compared to controls are paralleled by an absence of altered behavioral 294 responses. Thus, these two different markers support the lack of a long-term effect of juvenile 295 fluoxetine exposure on basal stress-related neuroendocrine responses and anxiety-like behavior. 296 In sum, juvenile fluoxetine treatment of zebrafish, at the dose and time tested, did not 297 alter adult expression of anxiety-like behavior or basal cortisol responses. This study provides a 298 basis for additional research that is necessary before any conclusions can be drawn regarding 299 permanent neuroadaptive changes elicited by juvenile exposure to fluoxetine. However, the 300 results of the current study suggest that, at least with regards to the basal regulation of stress 301 responses, juvenile fluoxetine exposure at this dose and time does not result in any unexpected or 302 detrimental effects in young adult zebrafish.

303

\section{Conclusions}

In the current study, juvenile zebrafish exposed to fluoxetine for 14 days did not display any alterations in basal cortisol levels or anxiety-like behavior in the novel tank test when assessed in young adulthood. These findings support the notion that SSRI exposure prior to sexual maturation has little effect on the basal regulation of neuroendocrine and behavioral markers of stress in adulthood. This study provides a foundation for additional research on the

310 impact of developmental SSRI exposure on adult markers. A complete understanding would 
311 require more extensive resources to fully clarify the impact of dose, targeted developmental

312 window, and differences between basal and stress-induced neuroendocrine and behavioral

313 responses.

\section{Acknowledgements}

315 Zebrafish embryos were obtained from the Zebrafish International Resource Center

316 (Eugene, OR). The use of Ethovision XT software was made possible by the Faculty for

317 Undergraduate Neuroscience (FUN) Equipment Loan program. The author thanks Dr. Jonathan

318 Karty of the Indiana University Mass Spectrometry Facility for assistance with some aspects of

319 this study. 
320

321

322

323

324

325

326

327

328

329

330

331

332

333

334

335

336

337

338

339

340

341

342

\section{References}

Abreu MS., Giacomini ACV., Koakoski G., Oliveira TA., Gusso D., Baldisserotto B., Barcellos LJG. 2015. Effects of waterborne fluoxetine on stress response and osmoregulation in zebrafish. Environmental toxicology and pharmacology 40:704-707.

Abreu MS., Giacomini ACVV., Koakoski G., Piato ALS., Barcellos LJG. 2017. Divergent effect of fluoxetine on the response to physical or chemical stressors in zebrafish. PeerJ 5:e3330.

Abreu MS de., Koakoski G., Ferreira D., Oliveira TA., Rosa JGS da., Gusso D., Giacomini ACV., Piato AL., Barcellos LJG. 2014. Diazepam and fluoxetine decrease the stress response in zebrafish. PloS one 9:e103232.

Airhart MJ., Lee DH., Wilson TD., Miller BE., Miller MN., Skalko RG. 2007. Movement disorders and neurochemical changes in zebrafish larvae after bath exposure to fluoxetine (PROZAC). Neurotoxicology and teratology 29:652-664.

Anderson SF., Kelley K., Maxwell SE. 2017. Sample-Size Planning for More Accurate Statistical Power: A Method Adjusting Sample Effect Sizes for Publication Bias and Uncertainty. Psychological science 28:1547-1562.

Andrews, M. H., \& Matthews, S. G. (2004). Programming of the hypothalamo-pituitary-adrenal axis: serotonergic involvement. Stress, 7(1), 15-27.

Ansorge MS., Zhou M., Lira A., Hen R., Gingrich JA. 2004. Early-life blockade of the 5-HT transporter alters emotional behavior in adult mice. Science 306:879-881.

Antidepressant Medications for Children and Adolescents: Information for Parents and Caregivers. Available at https://www.nimh.nih.gov/health/topics/child-and-adolescentmental-health/antidepressant-medications-for-children-and-adolescents-information-for- 
344 Bostic JQ., Rubin DH., Prince J., Schlozman S. 2005. Treatment of depression in children and 345 adolescents. Journal of psychiatric practice 11:141-154.

346 Cachat J., Stewart A., Grossman L., Gaikwad S., Kadri F., Chung KM., Wu N., Wong K., Roy S., Suciu C., Goodspeed J., Elegante M., Bartels B., Elkhayat S., Tien D., Tan J., Denmark A., Gilder T., Kyzar E., Dileo J., Frank K., Chang K., Utterback E., Hart P., Kalueff AV. 2010. Measuring behavioral and endocrine responses to novelty stress in adult zebrafish. Nature protocols 5:1786-1799.

Canavello PR., Cachat JM., Beeson EC., Laffoon AL., Grimes C., Haymore WAM., Elegante MF., Bartels BK., Hart PC., Elkhayat SI., Tien DH., Mohnot S., Amri H., Kalueff AV. 2011. Measuring Endocrine (Cortisol) Responses of Zebrafish to Stress. In: Kalueff AV, Cachat JM eds. Zebrafish Neurobehavioral Protocols. Totowa, NJ: Humana Press, 135142.

Center for Behavioral Health Statistics and Quality. 2016. Key substance use and mental health indicators in the United States: Results from the 2015 National Survey on Drug Use and Health (HHS Publication No. SMA 16-4984, NSDUH Series H-51). Retrieved from http://www.samhsa.gov/data/

Cousins L., Goodyer IM. 2015. Antidepressants and the adolescent brain. Journal of psychopharmacology 29:545-555.

Crago J., Klaper R. 2018. Place-based screening of mixtures of dominant emerging contaminants measured in Lake Michigan using zebrafish embryo gene expression assay. Chemosphere 365 
366 Cunha V., Rodrigues P., Santos MM., Moradas-Ferreira P., Ferreira M. 2016. Danio rerio

367 embryos on Prozac $\square$ Effects on the detoxification mechanism and embryo development.

$368 \quad$ Aquatic toxicology 178:182-189.

369 Cunha V., Rodrigues P., Santos MM., Moradas-Ferreira P., Ferreira M. 2018. Fluoxetine

370 modulates the transcription of genes involved in serotonin, dopamine and adrenergic

371 signalling in zebrafish embryos. Chemosphere 191:954-961.

372

Davis DJ., Klug J., Hankins M., Doerr HM., Monticelli SR., Song A., Gillespie CH., Bryda EC. 2015. Effects of Clove Oil as a Euthanasia Agent on Blood Collection Efficiency and Serum Cortisol Levels in Danio rerio. Journal of the American Association for Laboratory Animal Science: JAALAS 54:564-567.

DePasquale C., Neuberger T., Hirrlinger AM., Braithwaite VA. 2016. The influence of complex 377 and threatening environments in early life on brain size and behaviour. Proceedings. Biological sciences / The Royal Society 283. DOI: 10.1098/rspb.2015.2564.

Egan RJ., Bergner CL., Hart PC., Cachat JM., Canavello PR., Elegante MF., Elkhayat SI., Bartels BK., Tien AK., Tien DH., Mohnot S., Beeson E., Glasgow E., Amri H., Zukowska Z., Kalueff AV. 2009. Understanding behavioral and physiological phenotypes of stress and anxiety in zebrafish. Behavioural brain research 205:38-44.

Gentile S. 2010. Antidepressant use in children and adolescents diagnosed with major depressive 384 disorder: what can we learn from published data? Reviews on recent clinical trials 5:6375.

Giacomini ACVV., Abreu MS., Giacomini LV., Siebel AM., Zimerman FF., Rambo CL., Mocelin R., Bonan CD., Piato AL., Barcellos LJG. 2016a. Fluoxetine and diazepam acutely modulate stress induced-behavior. Behavioural brain research 296:301-310. 
389 Giacomini ACVV., Abreu MS., Zanandrea R., Saibt N., Friedrich MT., Koakoski G., Gusso D., 390 Piato AL., Barcellos LJG. 2016b. Environmental and Pharmacological Manipulations Blunt the Stress Response of Zebrafish in a Similar Manner. Scientific reports 6:28986.

392 Harper C \& Lawrence C. 2011. The Laboratory Zebrafish. Boca Raton, FL: CRC Press.

393 Hetrick SE., McKenzie JE., Cox GR., Simmons MB., Merry SN. 2012. Newer generation antidepressants for depressive disorders in children and adolescents. Cochrane database of systematic reviews 11:CD004851.

Iniguez SD., Alcantara LF., Warren BL., Riggs LM., Parise EM., Vialou V., Wright KN., Dayrit G., Nieto SJ., Wilkinson MB., Others. 2014. Fluoxetine exposure during adolescence alters responses to aversive stimuli in adulthood. Journal of Neuroscience 34:1007-1021.

Iñiguez SD., Warren BL., Bolaños-Guzmán CA. 2010. Short- and long-term functional consequences of fluoxetine exposure during adolescence in male rats. Biological psychiatry 67:1057-1066.

402

403

404

405

406

407

408

409

410

411

Kalichak F., Idalencio R., Rosa JGS., de Oliveira TA., Koakoski G., Gusso D., de Abreu MS., Giacomini ACV., Barcellos HHA., Fagundes M., Piato AL., Barcellos LJG. 2016. Waterborne psychoactive drugs impair the initial development of Zebrafish. Environmental toxicology and pharmacology 41:89-94.

Kalueff AV., Stewart AM., Gerlai R. 2014. Zebrafish as an emerging model for studying complex brain disorders. Trends in pharmacological sciences 35:63-75.

Kiryanova V., McAllister BB., Dyck RH. 2013. Long-term outcomes of developmental exposure to fluoxetine: a review of the animal literature. Developmental neuroscience 35:437-439.

Kodish I., Rockhill C., Varley C. 2011. Pharmacotherapy for anxiety disorders in children and adolescents. Dialogues in clinical neuroscience 13:439-452. 
412 Kwan W., Cortes M., Frost I., Esain V., Theodore LN., Liu SY., Budrow N., Goessling W., 413 North TE. 2016. The Central Nervous System Regulates Embryonic HSPC Production 414 via Stress-Responsive Glucocorticoid Receptor Signaling. Cell stem cell 19:370-382.

415 Maciag D., Simpson KL., Coppinger D., Lu Y., Wang Y., Lin RCS., Paul IA. 2006. Neonatal 416 antidepressant exposure has lasting effects on behavior and serotonin circuitry. 417 Neuropsychopharmacology: official publication of the American College of $418 \quad$ Neuropsychopharmacology 31:47-57.

419 Magno LDP., Fontes A., Gonçalves BMN., Gouveia A Jr. 2015. Pharmacological study of the 420 light/dark preference test in zebrafish (Danio rerio): Waterborne administration. Pharmacology, biochemistry, and behavior 135:169-176.

Marcon M., Herrmann AP., Mocelin R., Rambo CL., Koakoski G., Abreu MS., Conterato 423 GMM., Kist LW., Bogo MR., Zanatta L., Barcellos LJG., Piato AL. 2016. Prevention of unpredictable chronic stress-related phenomena in zebrafish exposed to bromazepam, fluoxetine and nortriptyline. Psychopharmacology 233:3815-3824.

Maximino C., Puty B., Benzecry R., Araújo J., Lima MG., de Jesus Oliveira Batista E., Renata de Matos Oliveira K., Crespo-Lopez ME., Herculano AM. 2013. Role of serotonin in zebrafish (Danio rerio) anxiety: Relationship with serotonin levels and effect of buspirone, WAY 100635, SB 224289, fluoxetine and para-chlorophenylalanine (pCPA) Diniz DLW., Gouveia A Jr., Oliveira KRM., Herculano AM. 2014. Fingerprinting of psychoactive drugs in zebrafish anxiety-like behaviors. PloS one 9:e103943. 
435 Maximino C., da Silva AWB., Gouveia A Jr., Herculano AM. 2011. Pharmacological analysis of 436 zebrafish (Danio rerio) scototaxis. Progress in neuro-psychopharmacology \& biological psychiatry 35:624-631.

438

439

440

441

442

443

444

445

446

447

448

449

450

451

452

453

454

455

456

457

Nesan D., Vijayan MM. 2013. Role of glucocorticoid in developmental programming: evidence from zebrafish. General and comparative endocrinology 181:35-44.

Norcross M., Mathur P., Poonam M., Enoch AJ., Karlsson R-M., Brigman JL., Cameron HA., Harvey-White J., Holmes A. 2008. Effects of adolescent fluoxetine treatment on fear-, anxiety- or stress-related behaviors in C57BL/6J or BALB/cJ mice. Psychopharmacology 200:413-424.

Oh J-E., Zupan B., Gross S., Toth M. 2009. Paradoxical anxiogenic response of juvenile mice to fluoxetine. Neuropsychopharmacology: official publication of the American College of Neuropsychopharmacology 34:2197-2207.

Park J-W., Heah TP., Gouffon JS., Henry TB., Sayler GS. 2012. Global gene expression in larval zebrafish (Danio rerio) exposed to selective serotonin reuptake inhibitors (fluoxetine and sertraline) reveals unique expression profiles and potential biomarkers of exposure. Environmental pollution 167:163-170.

Pavlidis M., Theodoridi A., Tsalafouta A. 2015. Neuroendocrine regulation of the stress response in adult zebrafish, Danio rerio. Progress in neuro-psychopharmacology \& biological psychiatry 60:121-131.

Pawluski JL., Rayen I., Niessen NA., Kristensen S., van Donkelaar EL., Balthazart J., Steinbusch HW., Charlier TD. 2012. Developmental fluoxetine exposure differentially alters central and peripheral measures of the HPA system in adolescent male and female offspring. Neuroscience 220:131-141. 
458 Pei S., Liu L., Zhong Z., Wang H., Lin S., Shang J. 2017. Erratum: Risk of prenatal depression 459 and stress treatment: alteration on serotonin system of offspring through exposure to $460 \quad$ Fluoxetine. Scientific reports 7:41344.

461 Pittman J., Hylton A. 2015. Behavioral, endocrine, and neuronal alterations in zebrafish (Danio 462 rerio) following sub-chronic coadministration of fluoxetine and ketamine. Pharmacology, biochemistry, and behavior 139 Pt B:158-162.

464

465

466

467 468

469

470

471

472

473

474

475

476

477

478

479

480

Pittman JT., Lott CS. 2014. Startle response memory and hippocampal changes in adult zebrafish pharmacologically-induced to exhibit anxiety/depression-like behaviors. Physiology \& behavior 123:174-179.

Rambo CL., Mocelin R., Marcon M., Villanova D., Koakoski G., de Abreu MS., Oliveira TA., Barcellos LJG., Piato AL., Bonan CD. 2017. Gender differences in aggression and cortisol levels in zebrafish subjected to unpredictable chronic stress. Physiology \& behavior 171:50-54.

Ramsay JM., Feist GW., Varga ZM., Westerfield M., Kent ML., Schreck CB. 2009. Whole-body cortisol response of zebrafish to acute net handling stress. Aquaculture 297:157-162.

Reeves RR., Ladner ME. 2010. Antidepressant-induced suicidality: An update. CNS neuroscience \& therapeutics 16:227-234.

Richendrfer H., Pelkowski SD., Colwill RM., Creton R. 2012. On the edge: pharmacological evidence for anxiety-related behavior in zebrafish larvae. Behavioural brain research 228:99-106.

Ryan ND. 2003. Child and adolescent depression: short-term treatment effectiveness and longterm opportunities. International journal of methods in psychiatric research 12:44-53.

Shrestha, S. S., Nelson, E. E., Liow, J. S., Gladding, R., Lyoo, C. H., Noble, P. L., ... \& Suomi, 
481

482

483

484

485

486

487

488

489

490

491

492

493

494

495

496

497

498

499

500

501

502 503

S. J. (2014). Fluoxetine administered to juvenile monkeys: effects on the serotonin transporter and behavior. American Journal of Psychiatry, 171(3), 323-331.

Steenbergen PJ., Richardson MK., Champagne DL. 2011. The use of the zebrafish model in stress research. Progress in neuro-psychopharmacology \& biological psychiatry $35: 1432-1451$.

Stewart AM., Braubach O., Spitsbergen J., Gerlai R., Kalueff AV. 2014. Zebrafish models for translational neuroscience research: from tank to bedside. Trends in neurosciences $37: 264-278$.

Sumpter JP., Donnachie RL., Johnson AC. 2014. The apparently very variable potency of the anti-depressant fluoxetine. Aquatic toxicology 151:57-60.

Theodoridi A., Tsalafouta A., Pavlidis M. 2017. Acute Exposure to Fluoxetine Alters Aggressive Behavior of Zebrafish and Expression of Genes Involved in Serotonergic System Regulation. Frontiers in neuroscience 11:223.

Tran S., Chatterjee D., Gerlai R. 2014. Acute net stressor increases whole-body cortisol levels without altering whole-brain monoamines in zebrafish. Behavioral neuroscience $128: 621-624$.

Vitiello B., Ordóñez AE. 2016. Pharmacological treatment of children and adolescents with depression. Expert opinion on pharmacotherapy 17:2273-2279.

Wendelaar Bonga SE. 1997. The stress response in fish. Physiological reviews 77:591-625.

Westerfield M. 2000. The zebrafish book: A guide for the laboratory use of zebrafish (Danio rerio), 4th ed. Eugene, OR: University of Oregon Press.

Wong RY., Oxendine SE., Godwin J. 2013. Behavioral and neurogenomic transcriptome changes in wild-derived zebrafish with fluoxetine treatment. BMC genomics 14:348. 


\section{Figure 1 (on next page)}

Trunk cortisol levels of young adult zebrafish treated during the juvenile period with and without fluoxetine.

Chronic fluoxetine treatment during the juvenile period (31-44 dpf) did not alter adult levels of trunk cortisol compared to control-treated fish ( $p=0.979$; independent samples t-test). Values are mean \pm SEM of 15-19 fish per group. 


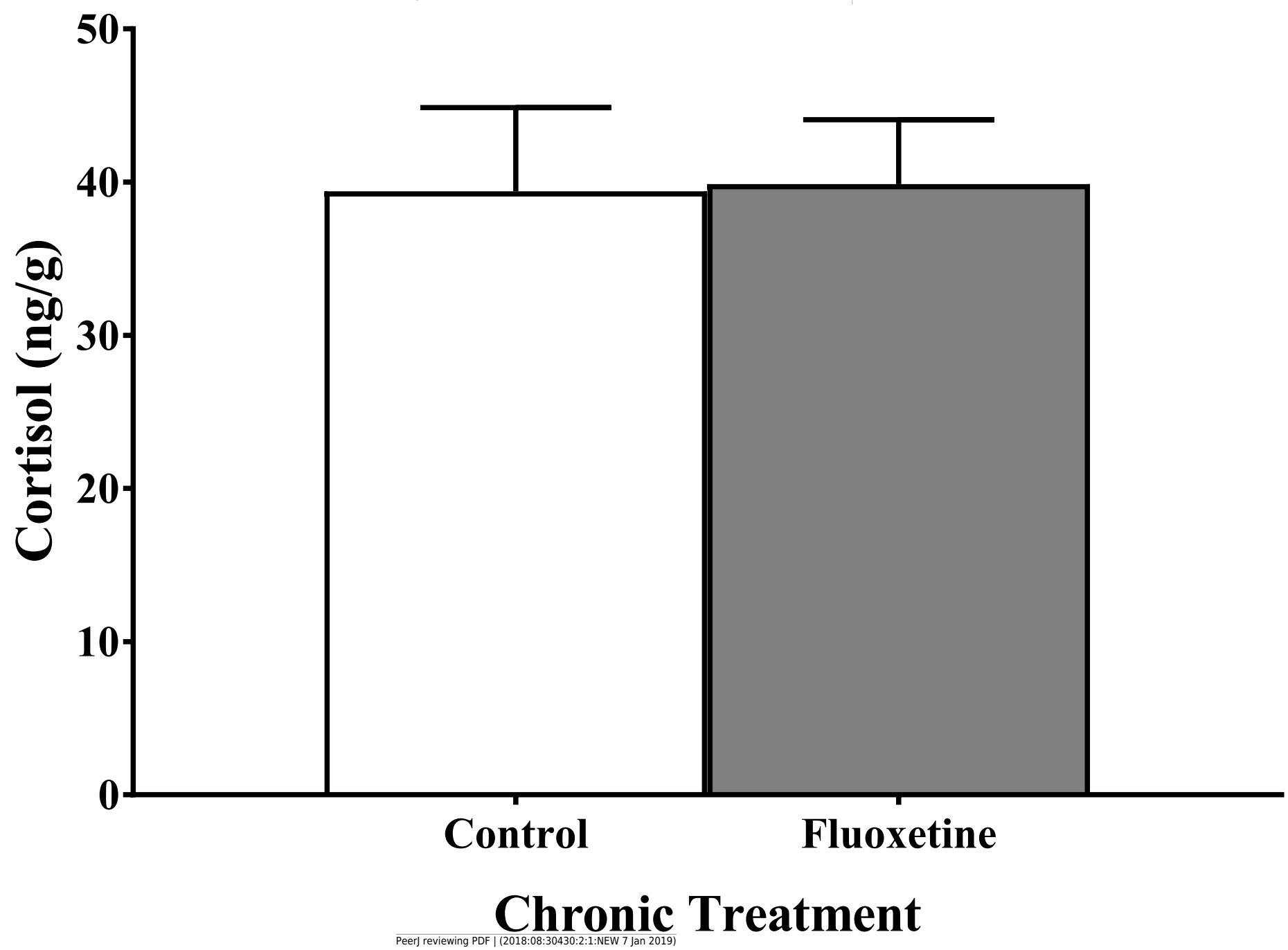




\section{Figure 2}

Motor activity measures of young adult zebrafish treated during the juvenile period with and without fluoxetine.

Chronic fluoxetine treatment during the juvenile period (31-44 dpf) did not alter the (A) total distance ( $p=0.303$; independent samples t-test) or (B) mean speed ( $p=0.594$; independent samples t-test) of adult fish in the novel tank test compared to control-treated fish. Values are mean \pm SEM of $12-13$ fish per group.

A

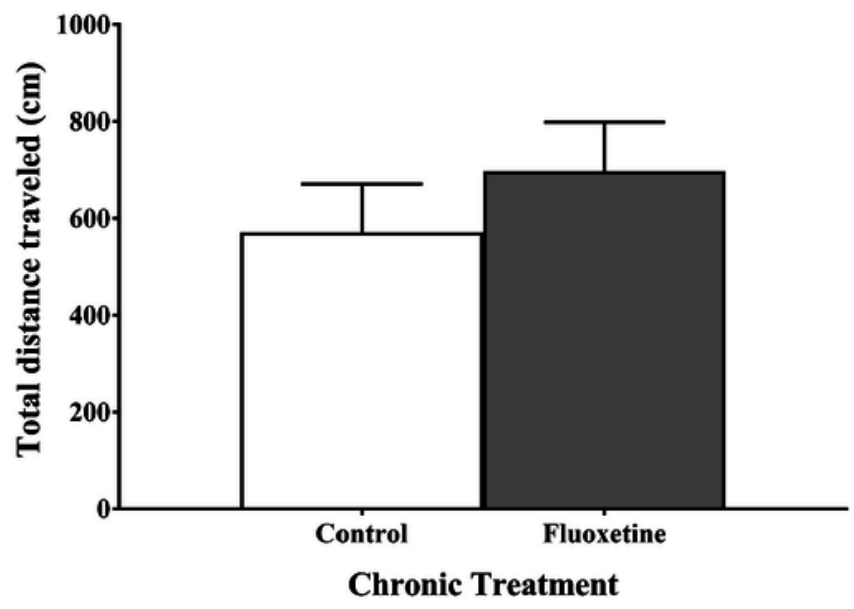

B

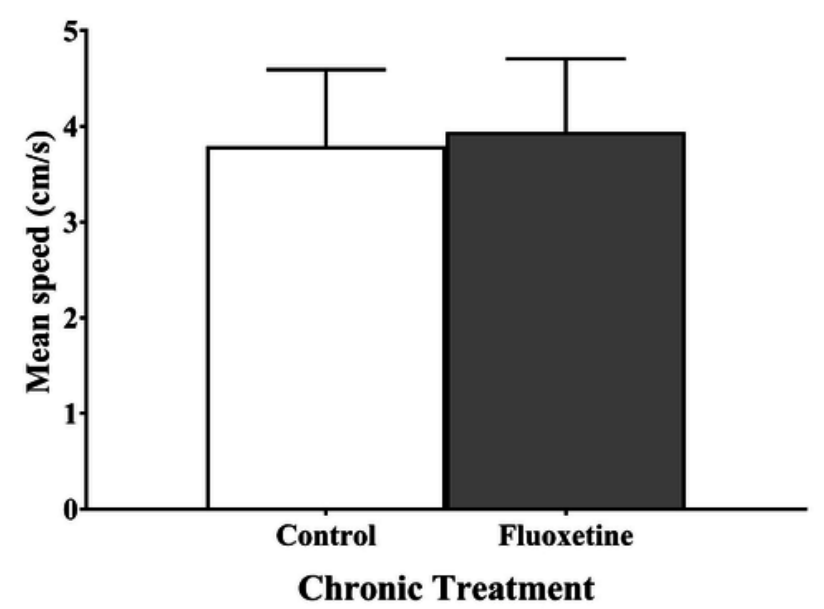




\section{Figure 3}

Freezing behaviors of young adult zebrafish treated during the juvenile period with and without fluoxetine.

Chronic fluoxetine treatment during the juvenile period (31-44 dpf) did not alter the (A) number of times immobile ( $p=0.634$; independent samples t-test) or $(B)$ total time immobile ( $p=0.595$; independent samples t-test) of adult fish in the novel tank test compared to control-treated fish. Values are mean \pm SEM of $12-13$ fish per group.

$\mathbf{A}$

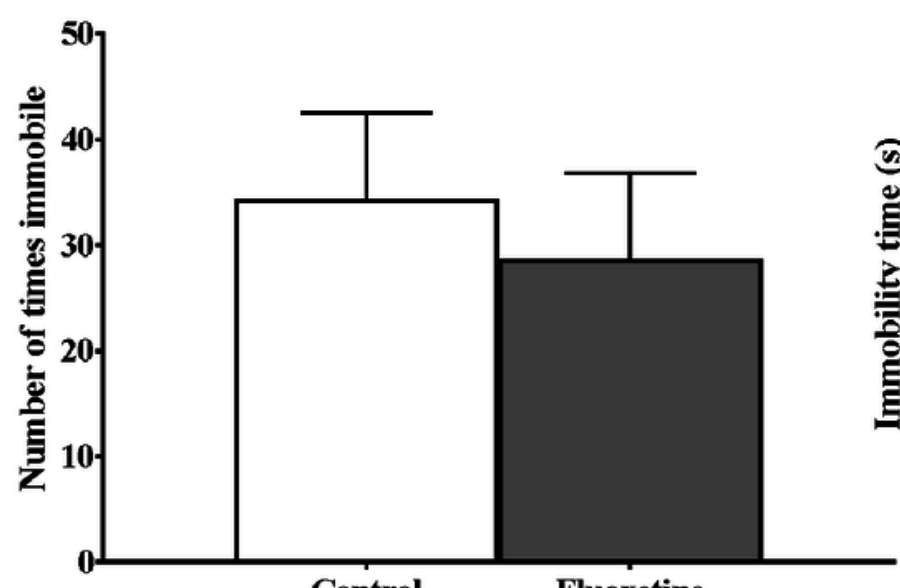

Control

B

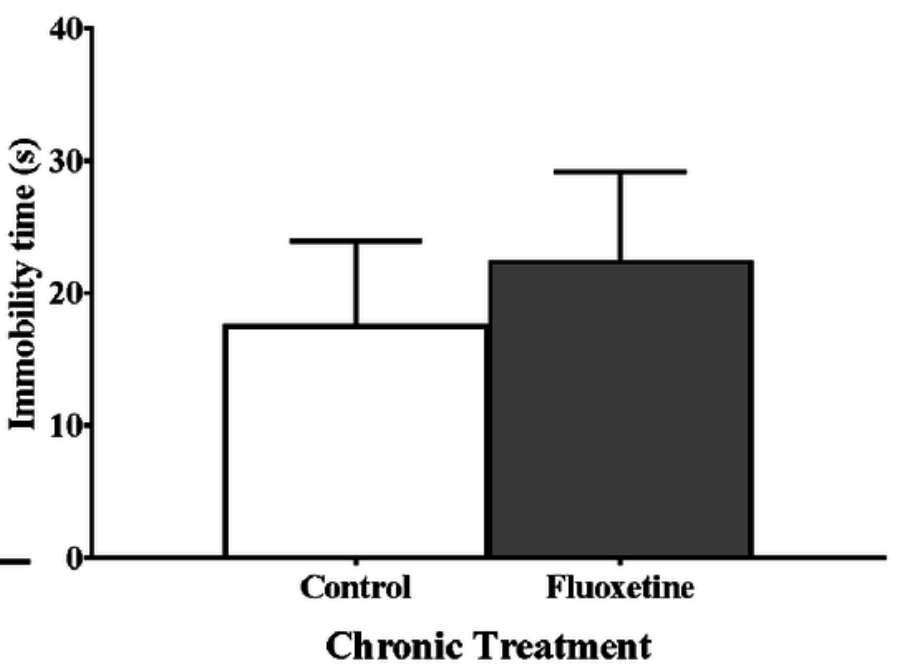




\section{Figure 4}

Anxiety-like behaviors of young adult zebrafish treated during the juvenile period with and without fluoxetine.

Chronic fluoxetine treatment during the juvenile period (31-44 dpf) did not alter the (A) distance in top ( $p=0.710$; independent samples t-test), (B) number of entries to top ( $p=$ 0.847 ; independent samples t-test), $(C)$ time in top ( $p=0.622$; independent samples t-test), or (D) latency to top ( $p=0.984$; independent samples t-test) of adult fish in the novel tank test compared to control-treated fish. Values are mean \pm SEM of 12-13 fish per group.

$\mathbf{A}$

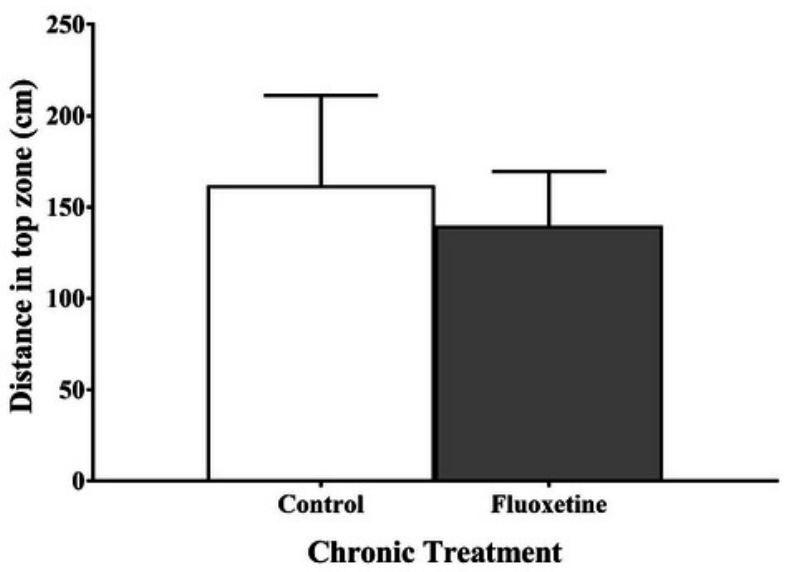

C

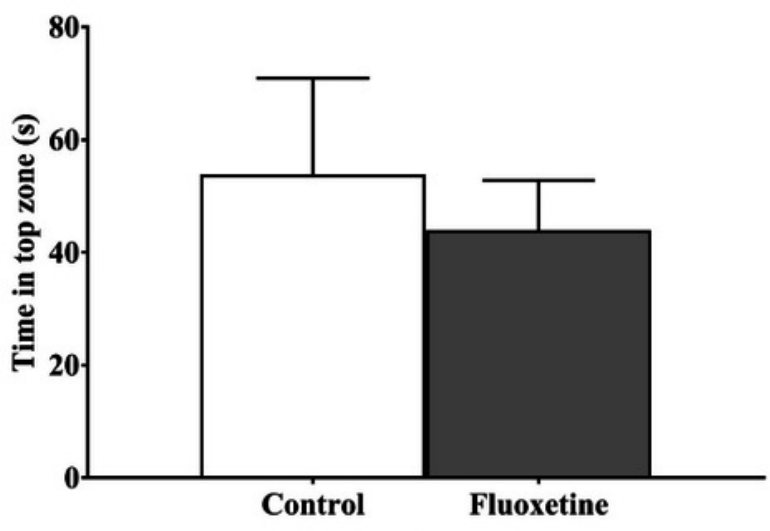

Chronic Treatment
B

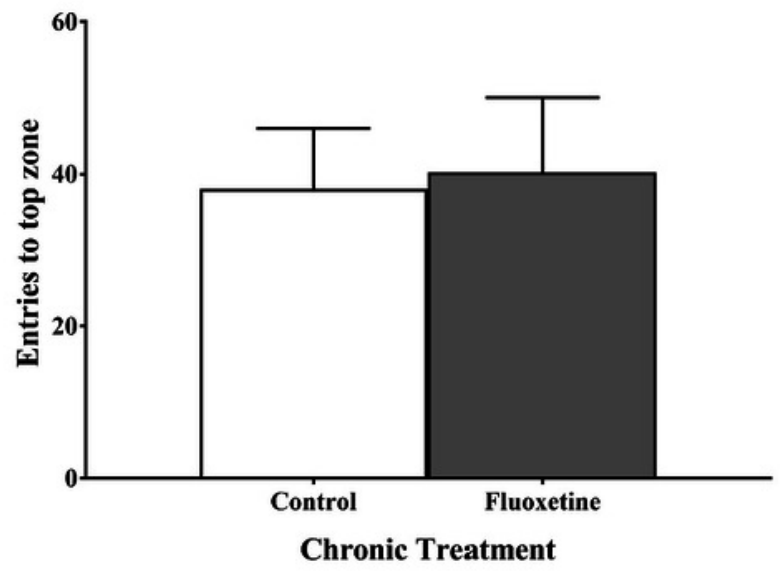

D

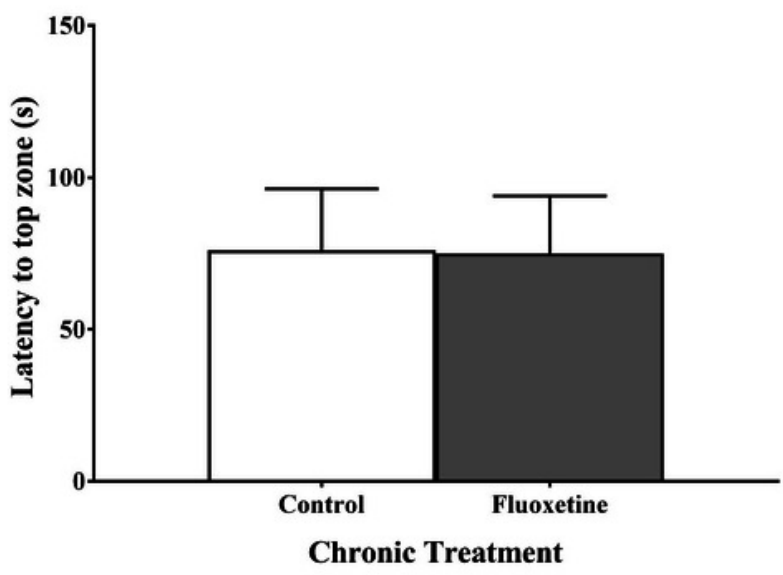

